\title{
Nonphysician Providers in Hospital Medicine: Not So Fast
}

Vikas I. Parekh, mo ${ }^{1}$ Christopher L. Roy, $\mathrm{mD}^{2}$

\author{
${ }^{1}$ Department of Internal Medicine, University of Michigan, Ann Arbor, Michigan. \\ ${ }^{2}$ Department of Medicine, Brigham and Women's Hospital, Boston, Massachusetts.
}

Disclosure: Nothing to report.

Ford and Britting's ${ }^{1}$ editorial in this month's Journal of Hospital Medicine raises important questions concerning the use of nonphysician providers in hospital medicine. They focus primarily on the use of mid-level providers (MLPs), namely physician-assistants (PAs) and nurse practitioners (NPs), as a potential solution to the current physician workforce shortages in our field. While we acknowledge the challenges of meeting workforce needs, we also believe that much is unknown about the optimal use of MLPs on inpatient general medicine services and it is premature to tout MLPs as the solution to hospital medicine staffing problems. This is especially true in those hospitals where hospitalists care for complex, general medical patients with a wide variety of medical conditions, a trend that is especially common in academic medical centers. ${ }^{2}$

This article discusses the current literature, our own experiences with MLPs, and suggests some future initiatives that might help better integrate MLPs into hospital medicine.

\section{The Literature on MLPs in Inpatient Venues}

The existing literature on the use of MLPs in inpatient venues is quite limited, and a recent review, while suggesting that the existing literature does describe benefits of MLPs in the inpatient setting, also states that the overall quality of the evidence is quite poor and that many studies suffer from significant limitations, including small populations, limited patient mixes, use of selected settings, and short durations of outcome assessment. ${ }^{3}$

Ford and Britting, ${ }^{1}$ in their article, cite several studies ${ }^{4-6}$ as evidence that a MLP model of care either improved outcomes or provided cost benefits. Each of these studies has important limitations that are worth examining.

The study by Myers et al. ${ }^{4}$ described the use of MLPs in a chest pain unit. NPs partnered with hospitalists to care for a low-acuity chest pain population. In addition, 5 NPs only staffed the unit during daytime weekday hours. Off-hour and weekend staffing was accomplished through the use of resident physicians. Notably, the work suggests the service only admitted 113 low-risk patients over 10 months. The service was staffed by 3 full-time equivalent (FTE) NPs in addition to involving hospitalists during the day. It is not surprising, given the extremely low volume of patients coupled with a daytime-only focus, that this service showed efficiency gains. In addition, given the service was only staffed by NPs 40 hours a week and by resident physicians on nights and weekends, the true cost of such an intervention needs to take into account the full cost of $24 / 7$ coverage. In addition, the model of using residents to cover nonteaching patients is no longer permitted by the current Accreditation Council for Graduate Medical Education (ACGME) Internal Medicine Residency Requirements ${ }^{7}$ and thus implementation of a model such as this in 2009 would require alternative means of nighttime coverage.

The study by Nishimura et al., ${ }^{5}$ also describing the use of MLPs in cardiovascular care, has important caveats that make full assessment of the model impossible. The model describes the implementation of a care team consisting of an attending, a fellow, and MLPs to replace a traditional teaching team of an attending, senior resident, and 2 interns. The study states that the model resulted in a lower length of stay (LOS) and lower costs per case. Importantly, the new MLP-based team only admitted during the hours of 7 AM to 2 PM. The study does not fully describe the number of MLPs required nor does it fully describe the role of cardiovascular fellows in the model. The study does state that the cost savings offset the cost of the MLPs but it is not clear if this cost analysis took into account the cost of the fellow's daytime involvement or if it measured attending time required before and after the implementation of the new model. In addition, this model presumes the availability of other services to admit patients during afternoon and nighttime hours and so may not be generalizable to other settings.

The final study by Cowan et al. ${ }^{6}$ describes the addition of a NP, a hospitalist medical director, and daily multidisciplinary rounds to a traditional teaching service model. Importantly, the NP was not involved in the admission process nor were they the primary providers for day-to-day medical care but rather they focused on implementation of care protocols, multidisciplinary coordination of care and discharge planning, and postdischarge follow-up. In addition, the NP worked only weekdays for about 40 hours a week. It is not surprising that adding multiple additional resources to existing care models might provide benefits but this does not address any issues in terms of the workforce since the care in this model required a higher total input of providers than the usual care model being studied. Cost savings from such a model may make it cost-effective but it does not represent a workforce solution.

There have been other studies examining the use of MLPs in the inpatient setting in internal medicine. Some of these studies have suggested that MLP-based models result 
in equivalent outcomes and efficiency ${ }^{8-10}$ to traditional teaching or nonteaching physician-only models. There are 2 important caveats, however, that must be considered. The total resources required for such models may be quite high, especially taking into account the costs of 24/7 coverage and physician backup of the MLPs, and most importantly there is almost no literature that robustly examines ultimate clinical outcomes in these models. We do note that a recent study $^{11}$ did show a lower inpatient mortality rate over a 2year period of time after substituting a PA-hospitalist model for a traditional academic medicine residency model in a community hospital. Importantly, however, the new model also added 24/7 hospitalist physicians and night and weekend intensivists that were not present in the prior residency-based model. Thus, the lower mortality rate could be attributed to the addition of hospitalists or the more robust in-house physician coverage during off-hours rather than the use of MLPs.

Notably, while the evidence base in internal medicine is not robust, many studies have described successful use of MLPs in non-internal medicine inpatient settings. ${ }^{12-14}$ The reasons for this success is debatable, but it may be that MLPs are more successful in settings where the care is either more protocol-driven or where there is less diagnostic and therapeutic complexity.

\section{Recent Experiences with MLPs in Academic Hospital Medicine}

Given the paucity of data, it is clear that further research is needed on the role of MLPs in hospital medicine. While waiting for such evidence to appear, it may be worthwhile to reflect on the recent experience of 3 major medical centers. A recent article described 5 hospitalist models at major academic medical centers across the country. Two of the institutions described at the time (University of Michigan Health System, Ann Arbor, MI; and Brigham and Women's Hospital, Boston, MA) utilized MLPs as a major element of their staffing of nonresident hospitalist services while another (University of California, San Francisco [UCSF] Medical Center at Mt. Zion, San Francisco, CA) had previously used MLPs as part of its model but phased them out about 1 year prior to publication of the article. ${ }^{2}$ The model used by the Brigham and Women's Hospital was later described in more detail in a subsequent publication. ${ }^{8}$ Recently 1 of these institutions (Michigan) has chosen to phase out MLPs. At Michigan, a 4-year experience with PAs on a general-medicine focused hospitalist service eventually led to the conclusion that continued use of PAs was not cost-effective. Significant barriers to success included a steep learning curve and the significant time required before PAs developed sufficient autonomy and efficiency in caring for a highly complex heterogeneous patient population. In the Michigan experience, PAs took up to 2 years to attain a significant level of autonomy and efficiency and even then some PAs still required a significant amount of physician oversight. Similar concerns at UCSF Mt. Zion led to the elimination of their MLP program as well. At Brigham and Women's, the MLP service continues but has required additional hospitalist staffing due to difficulties recruiting qualified MLPs with appropriate inpatient experience. In all cases, the models were challenged by high costs and the difficulty of developing MLPs to attain the level of autonomy and efficiency needed to justify their continued use. A key point is that in each institution, MLPs continue to play an important role in some specialty inpatient areas such as Hematology/Oncology and Bone Marrow Transplant, which is where MLPs have traditionally found their niche in inpatient Internal Medicine. These "focus shops" allow MLPs to develop a niche and expertise in a specialized area, where they may become more autonomous and efficient than house staff. Thus these settings may be more appropriate for MLPs than a heterogeneous general medicine inpatient setting.

\section{Reviewing the Financial Case}

In their article, Ford and Britting ${ }^{1}$ cite potential financial advantages for the use of MLPs in hospital medicine by comparing the relative salaries of MLPs to Hospitalists. What was missing in their analysis was the relative productivity of the 2 types of providers. We do have some limited data from the Society of Hospital Medicine (SHM) annual survey that looks at MLPs in hospital medicine but, again, the number of respondents for most data elements is less than 70, making generalizability difficult. Nonetheless, the data suggest that MLPs in hospital medicine average about $60 \%$ to $75 \%$ of the productivity of a physician when measured by encounters, although there is wide variability depending on the employment model (academic vs. multispecialty group). ${ }^{15}$ Importantly, the existing data do not provide any measure of how much physician input is provided to these MLPs but we suspect that in most models there is some physician time and input. If we presume that the MLPs bill independently and collect $85 \%$ of the physician fee schedule for a Medicare population, then collections would be about $50 \%$ to $65 \%$ of a typical physician. Given that median total compensation including benefits from the SHM survey was $\$ 120,000$ for MLPs and $\$ 216,000$ for physicians-about a 55\% ratio-this would argue for potential financial neutrality when substituting MLPs for physicians in a 2:1 ratio but only if we presume they require no physician supervision, which in our own experience is not likely in a general medicine population. In an alternative model, in which the physician sees every patient with the MLP and the physician bills, one would need to see roughly $50 \%$ more patients to achieve a financially neutral situation. In our experience at our own institutions, this level of increased productivity was not achievable. It is important to note that our figures are median compensation and benefit cost figures and local markets vary widely. We know that in major east and west coast cities MLPs may command far higher salaries while early career hospitalist physicians may 
be paid somewhat less than the reported medians. Recent market changes have significantly pressured MLP salaries, ${ }^{15,16}$ further impacting the financial equation and perhaps tilting it farther against a financial benefit for MLPs. Furthermore, night coverage for MLP services should always be considered in a financial analysis and is not captured in this simple analysis.

\section{Next Steps}

Given the current shortage of physicians, we imagine that many hospitalist groups will consider the use of MLPs as a solution to the current workforce issues. However, data on how best to utilize MLPs and the true impact on both the cost and quality of such models is lacking. In addition to urging increased publication and dissemination of existing experiences with NP and PAs, we strongly suggest that groups considering starting a MLP model do so in a way which would facilitate robust analysis and comparison of the model with alternatives. We also suggest that SHM consider the following: modifying its biennial survey to better capture the nuances of MLP productivity (such as assessing the amount of physician input and supervision required); targeting MLPs so as to increase the number of respondents; and doing an additional survey to capture demographics and basic data on existing MLP models given the lack of published literature.

In addition to gathering more data on effective models, a critical gap that we have identified is the development of models for the training and development of MLPs interested in hospital medicine. It would be a mistake to believe that MLPs could function in a manner similar to residencytrained physicians if they do not undergo similar training. NP/PA programs generally do not have a significant inpatient internal medicine focus and so newly minted graduates often lack the skills needed to succeed in hospital medicine. ${ }^{17}$ Some hospitalist programs train their MLPs on the job, but many programs cannot afford the amount of time and effort required to do this on their own. There are a small number of advanced training options for MLPs in hospital medicine ${ }^{18}$ but it is not likely such models will proliferate given the inherent opportunity costs that exist for extended training in the current competitive job market for MLPs. Instead we think that very motivated hospital medicine groups may develop training relationships with PA and NP schools in an effort to "train their own." In addition, national initiatives such as the Hospital Medicine Boot Camp for NPs and PAs, which is cosponsored by SHM, the American Association of Physician Assistants (AAPA), and the American Academy of Nurse Practitioners (AANP), ${ }^{19}$ can help fill the educational needs for MLPs who are already in practice.

\section{Conclusions}

While some literature exists that suggests that MLPs can successfully be used in the inpatient internal medicine set- ting, it is important to note that the evidence is quite limited and cannot be generalized across all care settings and patient populations. There is an urgent need to gather more data and share our collective experiences to better inform our decision-making before we state that MLPs are the solution to workforce shortages in hospital medicine. In addition, existing data and experience suggest that MLPs may not be a cost-effective workforce solution for complex general medical patients who require significant physician input. We believe that redesigning the clinical training of MLPs to focus on inpatient skills may hold promise and encourage interested parties to consider developing partnerships with MLP training programs and hospital medicine groups, as a way to build a more robust and successful hospital medicine MLP workforce.

\section{Address for correspondence and reprint requests:}

Vikas I. Parekh, MD, Associate Director, Hospitalist Program, Department of Internal Medicine, University of Michigan Medical School, 3116 Taubman Center SPC 5376, 1500 East Medical Center Drive, Ann Arbor, MI 48109-0376; Telephone: 734-647-2892; Fax: 734-615-8401; E-mail: viparekh@umich.edu Received 24 April 2009; revision received 26 May 2009; accepted 21 June 2009.

\section{References}

1. Ford WT, Britting LL. Nonphysician providers in the hospitalist model: a prescription for change and a warning about unintended side effects. $J$ Hosp Med. 2010;5:99-102.

2. Sehgal N, Shah H, Parekh V, Roy C, Williams M. Non-housestaff medicine services in academic medical centers: models and challenges. $J$ Hosp Med. 2008;3:247-255.

3. Kleinpell R, Ely E, Grabenkort R. Nurse practitioners and physician assistants in the intensive care unit: an evidence-based review. Crit Care Med. 2008;36:2888-2897.

4. Myers J, Bellini L, Rohrbach J. Improving resource utilization in a teaching hospital: development of a nonteaching service for chest pain admissions. Acad Med. 2006;81:432-435.

5. Nishimura RA, Linderbaum JA, Naessens JM, Spurrier B, Koch MB, Gaines KA. A nonresident cardiovascular inpatient service improves residents' experiences in an academic medical center: a new model to meet the challenges of the new millennium. Acad Med. 2004;79;426-431.

6. Cowan MJ. The effect of a multidisciplinary hospitalist/physician and advance practice nurse collaboration on hospital care. J Nurs Adm. 2006; 36:79-85.

7. Accreditation Council for Graduate Medical Education. ACGME Program Requirements for Residency Education in Internal Medicine. Available at: http://www.acgme.org/acWebsite/downloads/RRC_progReq/140_internal_ medicine_07012009.pdf. Accessed July 2009.

8. Roy C, Liang CL, Lund M, et al. Implementation of a physician assistant/ hospitalist service in an academic medical center: impact on efficiency and patient outcomes. J Hosp Med. 2008;3:361-368.

9. Van Rhee J, Ritchie J, Eward AM. Resource use by physician assistant services versus teaching services. JAAPA. 2002;15:33-38.

10. Pioro MH, Landefeld CS, Brennan PF, Daly B, et al. Outcomes-based trial of an inpatient nurse practitioner service for general medical patients. $J$ Eval Clin Pract. 2001;7:21-33.

11. Dhuper S, Choksi S. Replacing an academic internal medicine residency program with a physician assistant-hospitalist model: a comparative analysis study. Am J Med Qual. 2009;2:132-139.

12. Reines H, Robinson L, Duggan M, O'Brien M, Aulenbach K. Integrating midlevel practitioners into a teaching service. Am J Surg. 2006;1:119-124.

13. Christmas AB, Reynolds J, Hodges S, et al. Physician extenders impact trauma systems. J Trauma. 2005;58(5):917-920.

2010 Society of Hospital Medicine DOI 10.1002/jhm.584 Published online in wiley InterScience (www.interscience.wiley.com). 
14. Thourani VH, Miller JI. Physician assistants in cardiothoracic surgery: a 30year experience in a university center. Ann Thorac Surg. 2006;1:195-199.

15. 2007-2008 Society of Hospital Medicine Bi-Annual Survey: the Authoritative Source on the State of the Hospital Medicine Movement. Philadelphia: Society of Hospital Medicine; 2008.

16. American Association of Physician Assistants. Physician Assistant Income. Available at: http://www.aapa.org/images/stories/iu08incchange. pdf. Accessed July 2009.

17. Accreditation Review Commission on Education for the Physician Assistant. Accreditation Standards for Physician Assistant Education, 3rd ed.
Available at: http://www.arcpa.org/Standards/3rdeditionwithPDchangesandregionals4.24.08a.pdf. Accessed July 2009.

18. Association of Postgraduate PA Programs. Postgraduate PA Program Listing by State. Available at: http://www.appap.org/index1.html. Accessed July 2009.

19. American Association of Physician Assistants. Adult Hospitalist Physician Assistant and Nurse Practitioner Boot Camp. Available at: http://www. aapa.org/component/content/article/23--general-/673-adult-hospitalistphysician-assistant-and-nurse-practitioner-boot-camp. Accessed July 2009 . 